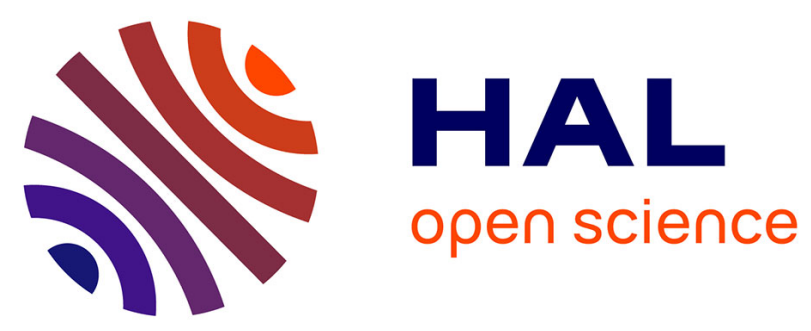

\title{
Liver contrast-enhanced sonography: Computer-assisted differentiation between focal nodular hyperplasia and inflammatory hepatocellular adenoma by reference to microbubble transport patterns
}

Baudouin Denis de Senneville, Nora Frulio, Hervé Laumonier, Cécile Salut, Luc Lafitte, Hervé Trillaud

\section{To cite this version:}

Baudouin Denis de Senneville, Nora Frulio, Hervé Laumonier, Cécile Salut, Luc Lafitte, et al.. Liver contrast-enhanced sonography: Computer-assisted differentiation between focal nodular hyperplasia and inflammatory hepatocellular adenoma by reference to microbubble transport patterns. European Radiology, 2020, 10.1007/s00330-019-06566-1 . hal-02377657v2

\author{
HAL Id: hal-02377657 \\ https://hal.science/hal-02377657v2
}

Submitted on 16 Nov 2020

HAL is a multi-disciplinary open access archive for the deposit and dissemination of scientific research documents, whether they are published or not. The documents may come from teaching and research institutions in France or abroad, or from public or private research centers.
L'archive ouverte pluridisciplinaire HAL, est destinée au dépôt et à la diffusion de documents scientifiques de niveau recherche, publiés ou non, émanant des établissements d'enseignement et de recherche français ou étrangers, des laboratoires publics ou privés. 


\section{Liver contrast-enhanced sonography: Computer-assisted}

\section{differentiation between focal nodular hyperplasia and}

\section{inflammatory hepatocellular adenoma by reference to}

\section{microbubble transport patterns}

Authors:

Baudouin Denis de Senneville ${ }^{1}, \mathrm{PhD}$, b.desenneville@gmail.com

Nora Frulio², MD, nora.frulio@ chu-bordeaux.fr

Hervé Laumonier ${ }^{2}, \mathrm{PhD}$, herve.laumonier@gmail.com

Cécile Salut ${ }^{2}, \mathrm{MD}$, cecile.salut@chu-bordeaux.fr

Luc Lafitte ${ }^{1}$, MD, luclafitte@gmail.com

Hervé Trillaud ${ }^{2,3}, \mathrm{PhD}$, herve.trillaud@ chu-bordeaux.fr

\section{$\underline{\text { Affiliations }}$}

${ }^{1}$ Institut de Mathématiques de Bordeaux (IMB), UMR 5251 CNRS/Université de Bordeaux, 351 cours de la Libération, F-33405, Talence, France

${ }^{2} \mathrm{CHU}$ de Bordeaux, Service d'imagerie diagnostique et Interventionnelle Magellan/Saint André, F33000 Bordeaux, France

${ }^{3}$ EA IMOTION (Imagerie moléculaire et thérapies innovantes en oncologie), Université de Bordeaux, F-33000 Bordeaux, France

\section{Corresponding Author:}

Baudouin Denis de Senneville

Address: Institut de Mathématiques de Bordeaux (IMB), UMR 5251 CNRS/Université de Bordeaux, 351 cours de la Libération, F-33405, Talence, France

Phone: (+33) (0)5 40002592

Fax: (+33) (0)5 40002123

E-mail: $\underline{\text { b.desenneville@gmail.com }}$ 


\section{ABSTRACT}

Objective: A new computer tool is proposed to distinguish between focal nodular hyperplasia (FNH) and an inflammatory hepatocellular adenoma (I-HCA) using contrast-enhanced ultrasound (CEUS). The new method was compared with the usual qualitative analysis.

Methods: The proposed tool embeds an "optical flow" algorithm, designed to mimic the human visual perception of object transport in image series, to quantitatively analyse apparent microbubble transport parameters visible on CEUS. Qualitative (visual) and quantitative (computer-assisted) CEUS data were compared in a cohort of adult patients with either FNH or I-HCA based on pathological and radiological results. For quantitative analysis, several computer-assisted classification models were tested and subjected to cross-validation. The accuracies, area under the receiver-operating characteristic curve (AUROC), sensitivity and specificity, positive predictive values (PPVs), negative predictive values (NPVs), false predictive rate (FPRs) and false negative rate (FNRs) were recorded.

Results: Forty-six patients with FNH $(n=29)$ or I-HCA $(n=17)$ with 47 tumors (one patient with 2 I-HCA) were analysed. The qualitative diagnostic parameters were: accuracy $=93.6 \%$, AUROC $=0.94$, sensitivity $=94.4 \%$, specificity $=93.1 \%, \mathrm{PPV}=89.5 \%$ and $\mathrm{NPV}=96.4 \%, \mathrm{FPR}=$ $6.9 \%, \mathrm{FNR}=5.6 \%$. The quantitative diagnostic parameters were: accuracy $=95.9 \%$, AUROC $=$ 0.97, sensitivity $=93.4 \%$, specificity $=97.6 \%, \mathrm{PPV}=95.3 \%$, and NPV $=96.7 \%, \mathrm{FPR}=2.4 \%, \mathrm{FNR}$ $=6.6 \%$.

Conclusions: Microbubble transport patterns evident on CEUS are valuable diagnostic indicators. Machine-learning algorithms analysing such data facilitate the diagnosis of FNH and I-HCA tumours.

\section{Key Points:}

- Distinguishing between focal nodular hyperplasia and an inflammatory hepatocellular adenoma using dynamic contrast-enhanced ultrasound is sometimes difficult. 
- Microbubble transport patterns evident on contrast-enhanced sonography are valuable diagnostic indicators.

- Machine-learning algorithms analysing microbubble transport patterns facilitate the diagnosis of FNH and I-HCA.

- The technique offers a potential future means for accurately characterizing other hepatic lesions, potentially obviating the need for biopsy or surgical resection.

Keywords: Ultrasound Imaging ; Adenoma ; Perfusion imaging ; Computer-Assisted Diagnosis ; Retrospective studies.

\section{Abbreviations and acronyms:}

US: Ultrasound

CEUS: contrast-enhanced ultrasound

FNH: Focal nodular hyperplasias

HCA: Hepatocellular adenomas

I-HCA: Inflammatory hepatocellular adenoma

CT: Computed tomography

MRI: Magnetic resonance imaging

T: Tesla

CNIL: National Commission on Informatics and Liberty

RF: Random forest

KNN: k-nearest neighbour

SVM: Support vector machine

LR: Logistic regression

PPV: Positive predictive value

NPV: Negative predictive value

AUC: Area under the curve

ROC: Receiver-Operating-Characteristic 


\section{GB: Gigabit}

RAM: Random access memory

\section{INTRODUCTION}

Benign hepatocellular tumours are rare, constituting $10 \%$ of all hepatic tumours (1). Two large groups of benign hepatocellular tumours can be distinguished: reactive regenerative lesions (focal nodular hyperplasias [FNHs]), and tumoural lesions (hepatocellular adenomas [HCAs]). Both lesions are most common in young females (1). Diagnostic imaging is essential to guide treatment decisions, which range from no treatment to surgical resection or confirmatory biopsy. Traditionally, multiphase computed tomography (CT) or magnetic resonance imaging (MRI) has been used for detailed evaluation of hepatic lesions. However, the high-level radiation associated with multiphase CT and the limited accessibility of MRI have rendered dynamic contrast agent-enhanced ultrasound (CEUS) an attractive, safe, non-invasive, accurate, and economic tool for evaluating hepatic lesions (2)-(6). Although the appearance is not always typical in some cases, both FNH and HCA demonstrate typical, reproducible, arterial phase enhancement patterns on CEUS in most cases. The diagnostic criteria for FNH are a hyper-enhancing lesion in the arterial phase with rapid centrifugal filling from a central vessel, and radial vascular branches (the "spoke and wheel" sign) (2)(5) and also sustained enhancement in portal and late phase (7). HCAs constitute a heterogeneous group of tumours exhibiting multiple histological subtypes (inflammatory, with FNH1A or catenin gene mutations, or unclassified) (8). On CEUS, HCAs are hyper-enhancing in the arterial phase; the enhancement pattern commences peripherally and exhibits rapid centripetal filling; this pattern is characteristic of 86-90\% of all inflammatory HCAs (I-HCAs). Other HCA subtypes exhibit iso-vascularity or moderate hyper-vascularity, with mixed filling patterns in the arterial phase (2)(9). In clinical practice, it is essential to distinguish FNH from adenoma to ensure appropriate management. Confirmed FNHs are managed conservatively (with regular follow-up); HCAs require cessation of oral contraceptive use, (commonly) biopsy, and 
either surgery or (at least) follow-up imaging. I-HCA show the most important hypervascularity and $10-15 \%$ of I-HCA are also found to be $\beta$-catenin activated with a risk for malignant transformation. Distinguishing between FNH and I-HCA using CEUS is sometimes difficult because both lesions evidence hyper-enhancement during the arterial phase and it can be challenging to qualitatively differentiate centrifugal from centripetal tumour filling, particularly for larger nodules. Computerassisted methods are thus required for quantitative spatiotemporal assessment of organ perfusion. Such techniques must be faster and more reproducible than visual analysis, and must lack learning curves. Efforts have been made to quantify enhancement parameters in vascular compartments as indicators of several pathological conditions (10-14). In particular, transport equations have been recently derived to estimate microbubble velocity at the time of bolus contrast arrival (15). In practice, a so-called "optical flow" algorithm is employed to mimic the human visual perception of microbubble transport in CEUS (16-18). Here, we use this approach to quantitatively distinguish between FNH and I-HCA. We quantify divergence (sources and sinks), curling (shearing), amplitudes, and convergence towards the centre of tumour (centrifugal/centripetal nature) in dense transport fields (16); these are very simple indicators of displacement vector directions, orientations, and magnitudes. In turn, these serve as inputs to a binary FNH/I-HCA classifier.

The purpose was to compare, as a preliminary study, the original concept of computer-assisted method with the usual qualitative analysis for the diagnosis of two benign hepatocellular tumours (FNH and I-HCA) with hypervascularity during the arterial phase of the CEUS.

\section{MATERIALS AND METHODS}

\section{Study design and population}

In this retrospective single-centre study conducted from July 2005 to July 2018, we identified images from patients who underwent CEUS and were (otherwise) definitively diagnosed with FNH or I-HCA. We included I-HCA patients who had been histologically diagnosed (6) and FNH patients diagnosed based on commonly accepted MRI criteria (3), imaging follow-up, or 
histologically. All MRIs were performed using a 1.5-T machine running a published imaging protocol (19)(20). The study adhered to all local regulations and data protection agency recommendations (the National Commission on Informatics and Liberty (CNIL) dictates). Patients have been informed for the use of their data anonymously.

\section{Demographic characteristics}

We enrolled 46 patients (Table 1) with the inclusion criteria, 29 had FNH and 17 I-HCA (18 I-HCA tumours were analysed because one patient had two tumours). Of $29 \mathrm{FNH}$ patients, 23 (79\%) were female and the median age was 44 (21-61) years; of 17 I-HCA patients, 16 (94\%) were female and the median age was 40.5 (21-66) years. The median diameters of FNH and I-HCA lesions were respectively $2.9(3-10)$ and $6.9(3.4-12) \mathrm{cm}$. Histological data of the 18 I-HCA tumours were available for 15 surgical specimens and 3 percutaneous biopsies Histological data on 7/29 FNH tumours (24\%) were available (percutaneous biopsy, six samples; one surgical sample); imaging follow-up data were available for $15 / 22$ patients without histological diagnosis $(68 \%)$ with a median follow-up of 12 (4-84) months ; CEUS was performed using Sequoia ( $\mathrm{n}=37), \mathrm{S} 2000(\mathrm{n}=4)$, and S3000 ( $n=5)$ instruments.

\section{Histological analysis}

Histological samples were obtained by biopsy or during surgical resection; for ethical reasons, no samples were taken purely for the purpose of this study; clinical indications were required. All analyses were performed as previously described (8)(9)(19), in the same laboratory.

\section{CEUS protocol}

CEUS was performed by abdominal radiologists who had 5-10 years of experience. Each patient received a bolus injection of ultrasound contrast agent (SonoVue, Bracco). Contrast-enhanced sequences were obtained using dedicated, low mechanical index (MI) contrast-imaging software (MI < 0.2) employing one of three US machines (Sequoia, S2000 and S3000; a Siemens Medical Solution instrument featuring Cadence Contrast Pulse Sequencing [CPS]; and a Convex Array 4C1$\mathrm{S}$ probe). Standard pre-settings were used, but it was possible to adjust settings for individual 
patients. SonoVue was injected intravenously as a bolus of $2.4 \mathrm{~mL}$ via a 20 -gauge cannula into the antecubital vein, followed by flushing with $5 \mathrm{~mL}$ normal saline. Digital cine clips showing dynamic contrast enhancement within the lesion and surrounding liver tissue were continuously recorded, commencing $5 \mathrm{~s}$ before SonoVue injection and covering the arterial (10-45 s post-injection), portal (60-90 s), and late (120-150 s) phases. Injection was repeated using the same dose $(2.4 \mathrm{~mL}$ SonoVue) if the data were of poor quality. All sequences were digitally stored. Intra-tumoural vascular geometry and lesional enhancement patterns were evaluated.

\section{CEUS analysis of lesional type}

\section{Qualitative visual analysis}

Data were reviewed in consensus by two abdominal radiologists blinded to pathological and MRI diagnoses. Each lesion was classified using pre-defined criteria for FNH and I-HCA. For FNH, these were hyper-enhancement in the arterial phase, with rapid centrifugal filling; (usually) an obvious central vessel and radial vascular branches (especially in larger lesions; the "spoke and wheel" sign); and iso- or hyper-enhancement in the portal and venous phases, without washout. For I-HCA, the criteria were hyper-enhancement in the arterial phase, frequently accompanied by rapid centripetal filling; no radial vascular structure; and iso- or hyper-enhancement in the portal and venous phases, without washout (3) (9) (21).

\section{Computer-assisted quantitative analysis using a transport equation model}

Microbubble transport fields in lesions were estimated (using a transport equation) on a pixel-bypixel basis employing the so-called "optical flow" process (15). The "optical flow" problem has long been studied by vision scientists in efforts to analyse general visual motion in images of a moving target (16)(17). For each lesion, the absolute changes in four image-based displacement indicators were calculated: (i) the divergence $\delta$ (reflecting the presence of sources and sinks); (ii) the curl $\rho$ (reflecting local vortices); (iii) the amplitude $\gamma$ (reflecting the magnitude of apparent displacement); and, (iv) the centripetal nature $\tau$ (reflecting the flow field convergence towards the centre of tumour). The analysis was restricted to a region of interest, manually drawn on a high 
contrast CEUS image, encompassing the tumour. The analytical window size was fixed at $2 \mathrm{~s}$ commencing at the bolus arrival time, and thus covered the filling phase. The reader is referred to Appendix A for additional information on numerical resolution and implementation. All computerassisted analyses were blinded to pathological data.

\section{Statistical analysis}

The accuracies, area under the ROC curve (AUROC), sensitivity, specificity, positive predictive values (PPVs), negative predictive values (NPVs), false predictive rate (FPRs) and false negative rate (FNRs) of qualitative and quantitative analyses were recorded (we considered the diagnostic of an adenoma as a "positive case" in the scope of this study).

For quantitative analyses, using one of the four microbubble displacement indicators $(\delta, \rho, \gamma$ or $\tau)$ as an input feature, we developed machine learning models to differentiate between FNH and I-HCA. For this binary classification task, the following four machine learning algorithms were applied using the commercial software Matlab (@1994-2019 The MathWorks, Inc.)/“"Statistics and Machine Learning" toolbox: random forest (RF), k-nearest neighbour (KNN), support vector machine (SVM), and logistic regression (LR). Default hyperparameters in Matlab implementations were employed. We refer the interested reader to (22) (23) for additional information about abovementioned computer-assisted classification algorithms. We evaluated the diagnostic performances through self-validation (the complete 47-tumours set was used for both train and test samples) and through 10-fold-stratified cross-validation (the 47-tumours set was randomly partitioned into complementary $90 \%$-training and 10\%-test subsets). The cross-validation steps were repeated 100 times with shuffling of the folds and test metric averages calculated. We also compared the medians and interquartile ranges of all four indicators using the unpaired Mann-Whitney U-test. A p-value < 0.025 was considered to reflect statistical significance.

\section{RESULTS}

\section{Qualitative CEUS analysis}

FNH and I-HCA were correctly identified via qualitative CEUS in 27/29 and 17/18 tumours, 
respectively (accuracy $=93.6 \%$, AUROC $=0.94$, sensitivity $=94.4 \%$, specificity $=93.1 \%, \operatorname{PPV}=$ $89.5 \%, \mathrm{NPV}=96.4 \%, \mathrm{FPR}=6.9 \%, \mathrm{FNR}=5.6 \%$; Table 2 , first row $)$

\section{Quantitative CEUS analysis}

Figures 1 and 2 show typical microbubble transport fields as revealed by dynamic contrast imaging; one clip (Fig. 1) is from an FNH patient and the other (Fig. 2) from an I-HCA patient. Of the four tested transport indicators, divergence and centripetal indicators differed most significantly between the two populations (Mann-Whitney test, $p$-value $=2 \times 10^{-4}$ for divergence, and $1 \times 10^{-7}$ for centripetal indicator) (Figs. 3 and 4). The centripetal indicator served as a valuable binary classifier in all tested machine learning systems (Table 2). In particular, using the naïve Bayes classifier applied on the centripetal indicator, the diagnostic parameters were: accuracy $=95.9 \%$, AUROC $=$ 0.97 , sensitivity $=93.4 \%$, specificity $=97.6 \%, \mathrm{PPV}=95.3 \%, \mathrm{NPV}=96.7 \%, \mathrm{FPR}=2.4 \%, \mathrm{FNR}=$ $6.6 \%$ (in average over the 100 cross-validation steps, FNH and I-HCA were thus correctly identified in 28.3/29 and 16.8/18 tumours, respectively).

\section{DISCUSSION}

We show that the dense transport fields provide valuable kinetic information in CEUS time series; the results are more accurate than those of qualitative visual analysis. Using the qualitative analysis, one false negative case and two false positive cases were to deplore. Concerning the false negative case, the filling direction was difficult to determine visually. Concerning the two false positive cases, one tumour $(44 \mathrm{~mm})$ presented two feeding pedicles, and one $(22 \mathrm{~mm})$ underwent a too fast filling. For both FNH tumours it was also difficult to appreciate visually the centrifugal filling. A quantitative approach delivers reproducible results and minimises operator dependency, as visual interpretation of CEUS images lacks a learning curve when the process is automated. Our method deals with the intrinsic variations in spatio-temporal greys that are inevitable during dynamic imaging. This allows numerical access to visual perceptions of microbubble trajectories. We used four simple indicators $(\delta, \rho, \gamma$, and $\tau)$ of transport field direction/orientation and amplitude. The amplitude and curl indicators were not useful (Figs. 4b, c), whereas the divergence and centripetal 
indicators were (Fig. 4a, d). Best results were obtained using the indicator $\tau$ which best fits the initial centrifugal/centripetal tumour filling hypothesis (Figs. 4d). For its part, the divergence operator gave decent results. In theory, the divergence of any vector field is positive for sources (centrifugal trajectories) and negative for sinks (centripetal trajectories). The divergence operators were positive for both FNH and I-HCA data; bolus arrival manifested as one or several sources of microbubbles. However, for I-HCA lesions, the divergence operator was modulated by centripetal filling, whereas the divergence operator was enhanced by centrifugal filling in FNH patients.

Using our quantitative method, diagnosis is near-instantaneous once the region of interest (encompassing the tumour) is delineated. Although the duration of the temporal window for the analysis must be sufficient to cover the filling phase, $2 \mathrm{~s}$ was adequate; this is a great advantage, eliminating all long-term bias imparted by probe motion, and respiratory and other motion artefacts $(24)(25)$.

Several limitations of our work must be mentioned, particularly the small sample size. This was a single-centre retrospective study lacking an external validation cohort. Considering that only two categories of focal liver lesions were examined (FNH and I-HCA), an inherent overestimation of both qualitative and quantitative analysis has to be taken into account. Also, mean tumour diameter was significantly smaller in the FNH group, associated with recruitment bias: only patients with histological diagnoses obtained after surgical resection or via percutaneous biopsy were included in the I-HCA group. However, in our centre, when an I-HCA tumour is identified using MRI (26) or CEUS, a pathological analysis is performed only when the tumour diameter is $>3 \mathrm{~cm}$. Thus, the IHCA group featured only tumours that met this criterion, unlike the FNH group, for which tumours of all diameters (including small tumours) were evaluated. Also, the fact that any US artefacts can intrinsically be interpreted as "false" motions by the transport equation constitutes a major source of uncertainty. This may bias the microbubble estimations in transport fields, in turn affecting all four image-based indicators. This is also of concern when brief US "shadow" artefacts develop in obese patients (one of our cohort was obese and was constantly miss-classified by our quantitative 
approach due to poor image quality). Similarly, in-plane and/or out-of-plane organ motion within the image field-of-view must be no more than moderate. Please note that when organ motions are large or complex, it is possible to "pop" microbubbles on-line to virtually repeat the imaging session. Alternatively, image post-processing strategies may be valuable (24)(28) (Appendix A). Finally, manually drawn masks encompassing lesions must exclude adjacent feeding arterials; otherwise, the estimated displacement is likely to be calculated from the border to the centre of the tumour, compromising FNH diagnosis.

In conclusion, this proof-of-concept study indicates that microbubble displacements evident on CEUS can be used to efficiently diagnose FNH/I-HCA lesions. Machine learning allows for computer-assisted diagnoses. In the future, we will optimise the model (28), enrol larger patient cohorts, include other lesional features, and study other pathologies. 


\section{REFERENCES}

1. Cherqui D, Rahmouni A, Charlotte Fet al (1995) Management of focal nodular hyperplasia and hepatocellular adenoma in young women: a series of 41 patients with clinical, radiological, and pathological correlations. Hepatology 22(6):1674-81.

2. Burrowes DP, Medellin A, Harris AC, Milot L, Wilson SR (2017) Contrast-enhanced US Approach to the Diagnosis of Focal Liver Masses. RadioGraphics 37(5):1388-400.

3. Dioguardi Burgio M, Ronot M, Salvaggio G, Vilgrain V, Brancatelli G (2016) Imaging of Hepatic Focal Nodular Hyperplasia: Pictorial Review and Diagnostic Strategy. Semin Ultrasound CT MR 37(6):511-24.

4. Trillaud H, Bruel JM, Valette PJ et al (2009) Characterization of focal liver lesions with SonoVue-enhanced sonography: international multicenter-study in comparison to CT and MRI. World J Gastroenterol 15(30):3748-56.

5. Baranes L, Chiaradia M, Pigneur F et al (2013) Imaging benign hepatocellular tumors: atypical forms and diagnostic traps. Diagn Interv Imaging 94(7-8):677-95.

6. Onofrio M, Crosara S, DeRobertis R, Canestrini S, Pozzi Mucelli R (2015) Contrast Enhanced Ultrasound of Focal Liver Lesions. AJR 2015 205:56-66.

7. Bartolotta TV, Taibbi A, Matranga D, Malizia G, Lagalla R, Midiri M (2010) Hepatic focal nodular hyperplasia:contrast-enhanced ultrasound findings with emphasis on lesion size, depth and liver echogenicity. Eur Radiol 20:2248-2256.

8. Bioulac-Sage P, Rebouissou S, Thomas C, et al (2007) Hepatocellular adenoma subtype classification using molecular markers and immunohistochemistry. Hepatol Baltim $\mathrm{Md}$ 46(3):740-8.

9. Laumonier H, Cailliez H, Balabaud C et al (2012) Role of contrast-enhanced sonography in differentiation of subtypes of hepatocellular adenoma: correlation with MRI findings. AJR Am J Roentgenol 199(2):341-8.

10. Tranquart F, Mercier L, Frinking P, Gaud E, Arditi M (2012) Perfusion quantification in contrast-enhanced ultrasound (CEUS)-ready for research projects and routine clinical use. Ultraschall Med. 33(1):S31-8.

11. Rognin NG, Arditi M, Mercier L, et al (2009) Parametric imaging of dynamic vascular patterns of focal liver lesions in contrast-enhanced ultra-sound. IEEE Ultrasonics Symp Proc 1282-1285.

12. Dietrich CF, Averkiou MA, Correas JM, Lasau N, Leen E, Piscaglia F (2012) An EFSUMB introduction into dynamic contrast-enhanced ultrasound (DCE-US) for quantification of tumour perfusion. Ultraschall in Med 33:344-351.

13. Strouthos C, Lampaksis M, Sboros V, McNeilly A, Averkiou M (2010) Indicator dilution models for the quantification of microvascular blood flow with bolus administration of ultrasound contrast agents. IEEE UFFC 57(6):1296-1310.

14. Mischi M, Kuenen MPJ, Wijkstra H (2012) Angiogenesis imaging by spatiotemporal analysis of ultrasound contrast agent dispersion kinetics. IEEE Transactions on Ultrasonics, Ferroelectrics, and Frequency Control 59(4):621-629. 
15. Denis de Senneville B, Novell A, Arthuis C et al (2018) Development of a fluid dynamic model for quantitative contrast-enhanced ultrasound imaging, IEEE Transactions on Medical Imaging 37(2):372-383.

16. Corpetti E, Mémin E, Pérez P (2002) Dense estimation of fluid flows. IEEE Transactions on Pattern Analysis and Machine Intelligence 24(3):365-380.

17. Horn B, Schunk B (1981) Determining optical flow. Artificial Intelligence 17:185-203.

18. Zachiu C, Papadakis N, Ries M, Moonen CTW, Denis de Senneville B (2015) An improved optical flow tracking technique for real-time MR-guided beam therapies in moving organs. Physics in Medicine and Biology 60(23):9003.

19. Laumonier H, Bioulac-Sage P, Laurent C, Zucman-Rossi J, Balabaud C, Trillaud H (2008) Hepatocellular adenomas: magnetic resonance imaging features as a function of molecular pathological classification. Hepatol Baltim Md 48(3):808-18.

20. van Aalten SM, Thomeer MGJ, Terkivatan $\mathrm{T}$ et al (2011) Hepatocellular adenomas: correlation of MR imaging findings with pathologic subtype classification. Radiology 261(1):172-81.

21. Quaia E, Calliada F, Bertolotto M et al (2004) Characterization of focal liver lesions with contrast-specific US modes and a sulfur hexafluoride-filled microbubble contrast agent: diagnostic performance and confidence. Radiology 232(2):420-30.

22. Kohavi R (1995) A Study of Cross-Validation and Bootstrap for Accuracy Estimation and Model Selection, Morgan Kaufmann, 1137-1143.

23. Cantor, SB, Kattan, MW (2000) Determining the Area under the ROC Curve for a Binary Diagnostic Test. Medical Decision Making 20(4), 468-470.

24. De Luca V, Székely G, Tanner C (2015) Estimation of large-scale organ motion in B-mode ultrasound image sequences: A survey. Ultrasound Med Biol 41(12):3044-3062.

25. Pratikakis I, Barillot C, Hellier P, Memin E (2003) Robust multiscale deformable registration of 3d ultrasound images. International Journal of Image and Graphics 3(4):547-565.

26. Bise S, Frulio N, Hocquelet A et al (2018) New MRI features improve subtype classification of hepatocellular adenoma. European Radiology, https://doi.org/10.1007/s00330-018-5784-5

27. Cifor A, Risser L, Chung D, Anderson EM, Schnabel JA (2013) Hybrid feature-based diffeomorphic registration for tumor tracking in 2-D liver ultrasound images, IEEE Trans Med Imaging 32( 9):1647-1656.

28. Ackermann D, Schmitz G (2016) Detection and tracking of multiple microbubbles in ultrasound b-mode images. IEEE Transactions on Ultrasonics, Ferroelectrics, and Frequency Control 63(1):72-82.

29. Shapiro LG, Stockman GC (2001) Computer Vision. Prentice Hall 137-150.

30. Marquardt D (1963) An algorithm for least squares estimation on nonlinear parameters. SIAM Journal on Applied Mathematics. 11:431-441. 


\begin{tabular}{|c|c|c|c|c|}
\hline Characteristic & FNH & I-HCA & Total & $\begin{array}{c}\text { Statistical significance } \\
\text { (p-value) }\end{array}$ \\
\hline Age & $\begin{array}{l}44 \pm 11 \\
(21-61)\end{array}$ & $\begin{array}{l}40.5 \pm 11 \\
(21-66)\end{array}$ & $\begin{array}{l}42 \pm 11 \\
(21-66)\end{array}$ & $\begin{array}{l}\text { No } \\
(0.16)\end{array}$ \\
\hline $\begin{array}{l}\text { Gender } \\
(\mathrm{F} / \mathrm{M})\end{array}$ & $23 / 6$ & $16 / 1$ & $39 / 7$ & - \\
\hline Tumor size & $\begin{array}{c}29 \pm 16 \\
(13-100)\end{array}$ & $\begin{array}{c}60.5 \pm 29 \\
(34-126)\end{array}$ & $\begin{array}{c}36 \pm 28 \\
(13-126)\end{array}$ & $\begin{array}{c}\text { Yes } \\
\left(10^{-6}\right)\end{array}$ \\
\hline $\begin{array}{c}\text { Histological data } \\
\text { (available/not available) }\end{array}$ & $7 / 22$ & $16 / 1$ & $23 / 23$ & - \\
\hline
\end{tabular}

Table 1. Demographic characteristics. Median values of age and tumor size are shown with standard deviations and minimum-maximum intervals in parentheses. Statistical comparison of age and tumor size between FNH and I-HCA groups were performed using an unpaired Mann-Whitney U-test (last column), a p-value $<0.025$ was considered to reflect statistical significance. 


\begin{tabular}{|c|c|c|c|c|c|c|}
\hline Classifier & Accuracy & AUROC & Sensitivity & Specificity & PPV & NPV \\
\hline Qualitative analysis & 93.6 & 0.94 & 94.4 & 93.1 & 89.5 & 96.4 \\
\hline \multicolumn{7}{|l|}{ Divergence $(\delta)$} \\
\hline Logistic Regression & $\begin{array}{l}86.6 \pm 14.9 \\
(85.6-87.5)\end{array}$ & $\begin{array}{l}0.82 \pm 0.23 \\
(0.80-0.83)\end{array}$ & $\begin{array}{l}74.1 \pm 35.1 \\
(71.8-76.3)\end{array}$ & $\begin{array}{l}94.3 \pm 16.5 \\
(93.3-95.4)\end{array}$ & $\begin{array}{l}81.7 \pm 34.7 \\
(79.5-83.9)\end{array}$ & $\begin{array}{l}87.9 \pm 16.5 \\
(86.8-88.9)\end{array}$ \\
\hline $\begin{array}{l}\text { Support Vector } \\
\text { Machine }\end{array}$ & $\begin{array}{l}86.9 \pm 14.6 \\
(86.0-87.8)\end{array}$ & $\begin{array}{l}0.82 \pm 0.21 \\
(0.81-0.84)\end{array}$ & $\begin{array}{l}75.5 \pm 33.8 \\
(73.4-77.7)\end{array}$ & $\begin{array}{l}94.1 \pm 16.4 \\
(93.0-95.1)\end{array}$ & $\begin{array}{l}83.1 \pm 33.0 \\
(81.0-85.2)\end{array}$ & $\begin{array}{l}88.6 \pm 15.7 \\
(87.6-89.6)\end{array}$ \\
\hline Naive Bayes & $\begin{array}{l}86.6 \pm 14.8 \\
(85.7-87.6)\end{array}$ & $\begin{array}{l}0.81 \pm 0.23 \\
(0.80-0.83)\end{array}$ & $\begin{array}{c}74.9 \pm 34.6 \\
(72.7-77.1)\end{array}$ & $\begin{array}{l}94.1 \pm 16.2 \\
(93.0-95.1)\end{array}$ & $\begin{array}{l}82.0 \pm 34.0 \\
(79.8-84.1)\end{array}$ & $\begin{array}{l}88.1 \pm 16.5 \\
(87.0-89.1)\end{array}$ \\
\hline Random Forest & $\begin{array}{l}85.4 \pm 14.5 \\
(84.5-86.3)\end{array}$ & $\begin{array}{l}0.75 \pm 0.25 \\
(0.74-0.77)\end{array}$ & $\begin{array}{l}67.5 \pm 33.9 \\
(65.4-69.7)\end{array}$ & $\begin{array}{l}96.6 \pm 15.0 \\
(95.6-97.5)\end{array}$ & $\begin{array}{l}84.5 \pm 34.1 \\
(82.4-86.7)\end{array}$ & $\begin{array}{l}83.9 \pm 17.4 \\
(82.8-85.0)\end{array}$ \\
\hline \multicolumn{7}{|l|}{$\operatorname{Curl}(\rho)$} \\
\hline Logistic Regression & $\begin{array}{l}77.2 \pm 16.0 \\
(76.2-78.3)\end{array}$ & $\begin{array}{l}0.68 \pm 0.25 \\
(0.66-0.69)\end{array}$ & $\begin{array}{l}60.1 \pm 40.3 \\
(57.5-62.6)\end{array}$ & $\begin{array}{l}87.9 \pm 24.5 \\
(86.4-89.5)\end{array}$ & $\begin{array}{l}64.4 \pm 41.7 \\
(61.8-67.1)\end{array}$ & $\begin{array}{l}80.1 \pm 20.8 \\
(78.8-81.4)\end{array}$ \\
\hline $\begin{array}{l}\text { Support Vector } \\
\text { Machine }\end{array}$ & $\begin{array}{l}67.4 \pm 15.0 \\
(66.5-68.4)\end{array}$ & $\begin{array}{l}0.52 \pm 0.28 \\
(0.50-0.53)\end{array}$ & $\begin{array}{l}42.0 \pm 43.9 \\
(39.2-44.8)\end{array}$ & $\begin{array}{l}84.3 \pm 31.0 \\
(82.4-86.3)\end{array}$ & $\begin{array}{l}39.6 \pm 42.7 \\
(36.9-42.3)\end{array}$ & $\begin{array}{l}68.0 \pm 25.2 \\
(66.4-69.7)\end{array}$ \\
\hline Naive Bayes & $\begin{array}{l}76.7 \pm 16.3 \\
(75.6-77.7)\end{array}$ & $\begin{array}{l}0.69 \pm 0.25 \\
(0.67-0.71)\end{array}$ & $\begin{array}{l}60.2 \pm 41.5 \\
(57.6-62.9)\end{array}$ & $\begin{array}{l}87.0 \pm 24.8 \\
(85.4-88.6)\end{array}$ & $\begin{array}{l}61.7 \pm 41.9 \\
(59.0-64.4)\end{array}$ & $\begin{array}{l}80.4 \pm 21.0 \\
(79.1-81.8)\end{array}$ \\
\hline Random Forest & $\begin{array}{l}64.6 \pm 12.9 \\
(63.8-65.4)\end{array}$ & $\begin{array}{l}0.40 \pm 0.26 \\
(0.39-0.42)\end{array}$ & $\begin{array}{l}33.9 \pm 41.6 \\
(31.3-36.6)\end{array}$ & $\begin{array}{l}84.2 \pm 31.5 \\
(82.2-86.2)\end{array}$ & $\begin{array}{l}31.8 \pm 40.0 \\
(29.2-34.3)\end{array}$ & $\begin{array}{l}64.3 \pm 23.8 \\
(62.8-65.8)\end{array}$ \\
\hline \multicolumn{7}{|l|}{ Amplitude $(\gamma)$} \\
\hline Logistic Regression & $\begin{array}{l}72.2 \pm 15.9 \\
(71.2-73.3)\end{array}$ & $\begin{array}{l}0.56 \pm 0.28 \\
(0.55-0.58)\end{array}$ & $\begin{array}{l}48.8 \pm 42.4 \\
(46.1-51.5)\end{array}$ & $\begin{array}{l}86.9 \pm 27.4 \\
(85.1-88.6)\end{array}$ & $\begin{array}{l}52.5 \pm 44.4 \\
(49.7-55.3)\end{array}$ & $\begin{array}{l}74.1 \pm 22.7 \\
(72.7-75.6)\end{array}$ \\
\hline $\begin{array}{l}\text { Support Vector } \\
\text { Machines }\end{array}$ & $\begin{array}{l}67.2 \pm 14.7 \\
(66.3-68.2)\end{array}$ & $\begin{array}{l}0.52 \pm 0.27 \\
(0.50-0.53)\end{array}$ & $\begin{array}{l}40.6 \pm 44.1 \\
(37.8-43.4)\end{array}$ & $\begin{array}{l}83.9 \pm 31.0 \\
(81.9-85.9)\end{array}$ & $\begin{array}{l}37.8 \pm 41.9 \\
(35.2-40.5)\end{array}$ & $\begin{array}{l}68.5 \pm 25.0 \\
(66.9-70.1)\end{array}$ \\
\hline Naive Bayes & $\begin{array}{l}70.3 \pm 15.4 \\
(69.3-71.2)\end{array}$ & $\begin{array}{l}0.54 \pm 0.28 \\
(0.52-0.55)\end{array}$ & $\begin{array}{l}48.6 \pm 43.3 \\
(45.8-51.4)\end{array}$ & $\begin{array}{l}83.8 \pm 29.8 \\
(81.9-85.7)\end{array}$ & $\begin{array}{l}49.0 \pm 43.3 \\
(46.2-51.7)\end{array}$ & $\begin{array}{l}73.5 \pm 23.9 \\
(71.9-75.0)\end{array}$ \\
\hline Random Forest & $\begin{array}{l}80.2 \pm 16.2 \\
(79.2-81.3)\end{array}$ & $\begin{array}{l}0.70 \pm 0.28 \\
(0.69-0.72)\end{array}$ & $\begin{array}{l}61.6 \pm 38.6 \\
(59.1-64.0)\end{array}$ & $\begin{array}{l}91.9 \pm 20.9 \\
(90.6-93.3)\end{array}$ & $\begin{array}{l}71.4 \pm 40.7 \\
(68.8-74.0)\end{array}$ & $\begin{array}{l}80.8 \pm 20.0 \\
(79.5-82.1)\end{array}$ \\
\hline \multicolumn{7}{|l|}{ Centripetal indicator $(\gamma)$} \\
\hline Logistic Regression & $\begin{array}{l}95.7 \pm 10.1 \\
(95.1-96.3)\end{array}$ & $\begin{array}{l}0.97 \pm 0.09 \\
(0.96-0.97)\end{array}$ & $\begin{array}{l}93.5 \pm 19.6 \\
(92.3-94.8)\end{array}$ & $\begin{array}{l}97.1 \pm 11.2 \\
(96.4-97.8)\end{array}$ & $\begin{array}{l}94.9 \pm 17.7 \\
(93.8-96.0)\end{array}$ & $\begin{array}{l}96.8 \pm 10.3 \\
(96.1-97.4)\end{array}$ \\
\hline $\begin{array}{l}\text { Support Vector } \\
\text { Machines }\end{array}$ & $\begin{array}{l}95.8 \pm 10.0 \\
(95.1-96.4)\end{array}$ & $\begin{array}{l}0.97 \pm 0.09 \\
(0.96-0.97)\end{array}$ & $\begin{array}{l}92.8 \pm 21.3 \\
(91.4-94.1)\end{array}$ & $\begin{array}{c}97.5 \pm 9.4 \\
(97.0-98.1)\end{array}$ & $\begin{array}{l}94.6 \pm 19.1 \\
(93.4-95.8)\end{array}$ & $\begin{array}{r}96.7 \pm 9.9 \\
(96.1-97.3)\end{array}$ \\
\hline Naive Bayes & $\begin{array}{c}95.9 \pm 9.8 \\
(95.3-96.5)\end{array}$ & $\begin{array}{l}0.97 \pm 0.08 \\
(0.96-0.97)\end{array}$ & $\begin{array}{l}93.4 \pm 19.8 \\
(92.1-94.6)\end{array}$ & $\begin{array}{l}97.6 \pm 10.0 \\
(97.0-98.2)\end{array}$ & $\begin{array}{l}95.3 \pm 17.5 \\
(94.2-96.4)\end{array}$ & $\begin{array}{l}96.7 \pm 10.4 \\
(96.0-97.3)\end{array}$ \\
\hline Random Forest & $\begin{array}{l}91.8 \pm 11.9 \\
(91.0-92.5)\end{array}$ & $\begin{array}{l}0.92 \pm 0.13 \\
(0.91-0.92)\end{array}$ & $\begin{array}{l}83.4 \pm 28.0 \\
(81.6-85.2)\end{array}$ & $\begin{array}{l}97.0 \pm 10.5 \\
(96.3-97.7)\end{array}$ & $\begin{array}{l}91.5 \pm 24.3 \\
(89.9-93.0)\end{array}$ & $\begin{array}{l}92.4 \pm 12.6 \\
(91.6-93.2)\end{array}$ \\
\hline
\end{tabular}

Table 2. Diagnostic performances of the various classifiers. Qualitative (i.e., visual) and quantitative scores are given; the latter were derived via evaluation of divergence $\delta$, curl $\rho$, amplitude $\gamma$, and centripetal indicator $\tau$ (after 10-fold cross-validation) by various machine-learning algorithms. AUROC: area under the ROC curve; PPV: positive predictive value; NPV: negative predictive value. Accuracies, sensitivities, specificities, PPVs, and NPVs are shown in percentages. 
Quantitative indicators are shown with standard deviations and 95\% confidence intervals in parentheses. 


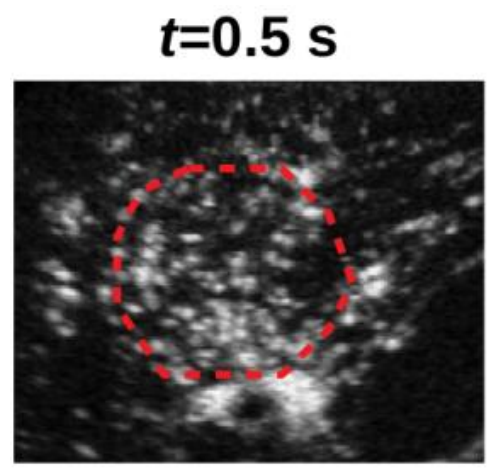

(a)

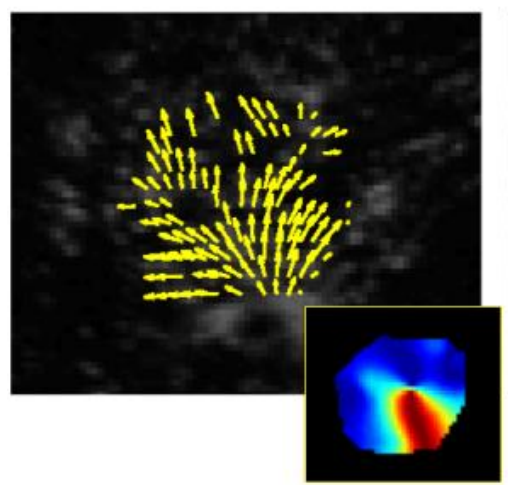

(d)

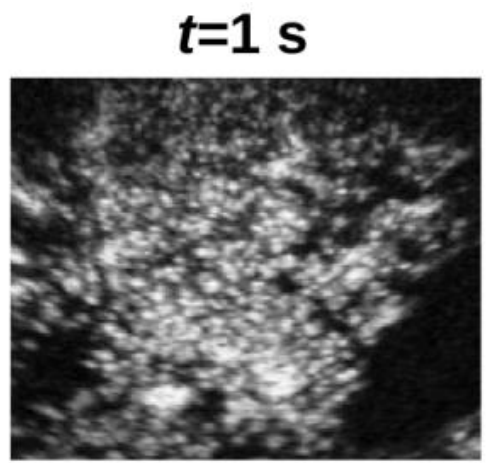

(b)

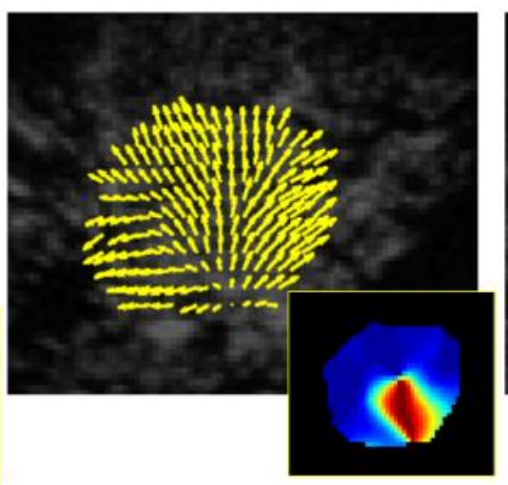

(e)

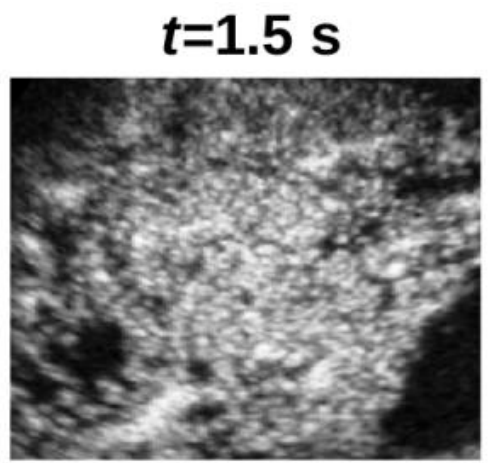

(c)

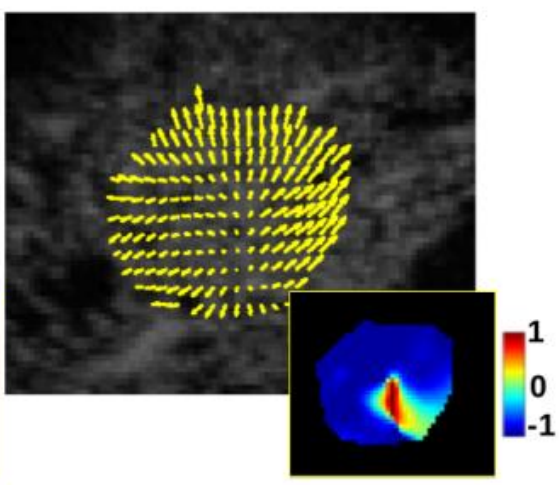

(f)

Fig. 1. Typical results obtained when evaluating an FNH lesion. Data obtained at different CEUS timepoints are shown: $0.5 \mathrm{~s}$ (left column), $1 \mathrm{~s}$ (middle column), and $1.5 \mathrm{~s}$ (right column) after bolus arrival. The manually drawn mask encompassing the lesion is shown in (a). Contrast images (top row) and estimated, apparent transport vector fields (bottom row). The flow field exhibits fast centrifugal filling of the lesion from a central vessel and radial vascular branches. The pixel-wise centripetal indicator is shown in the insets of the bottom row (note the large negative values, attributable to centrifugal filling of the lesion, and the small positive values attributable to the tumour feeding arterial). 


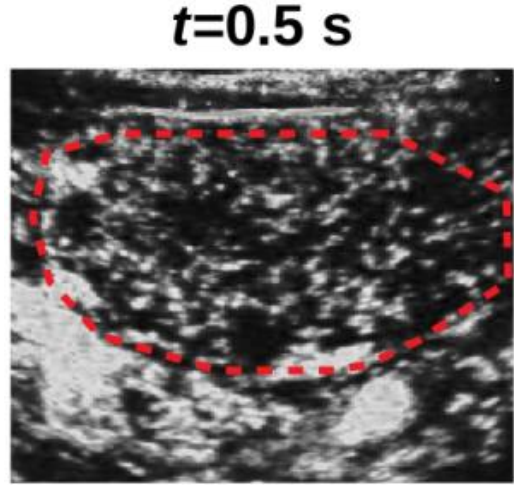

(a)

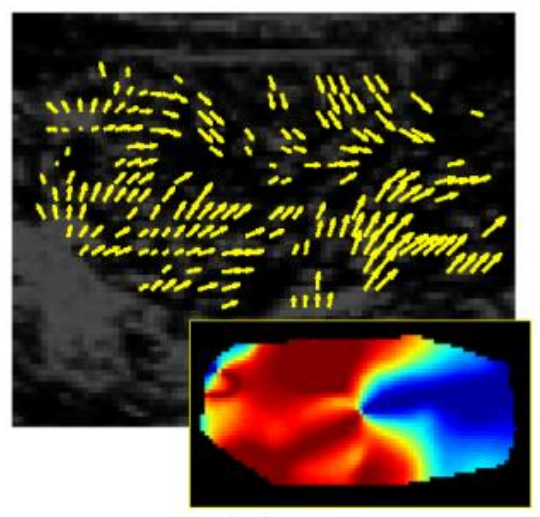

(d)

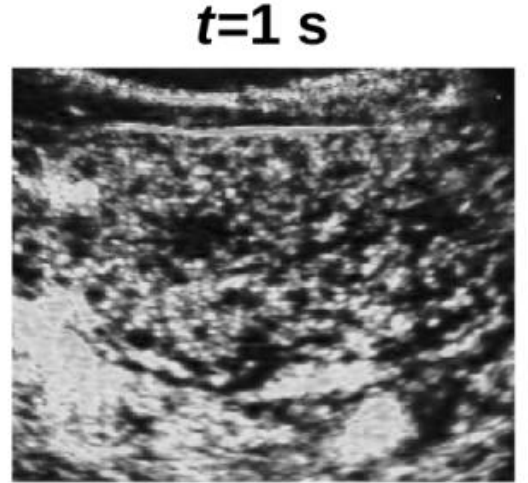

(b)

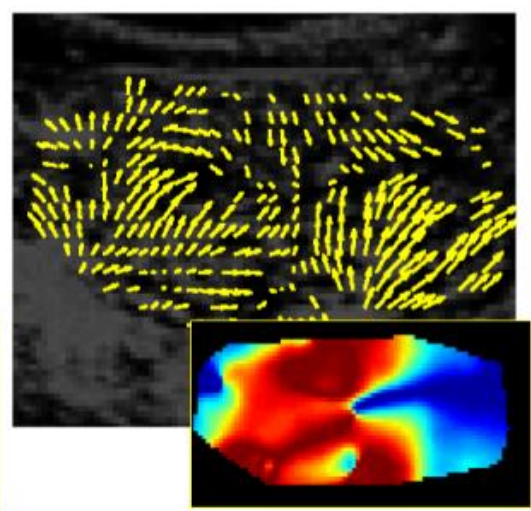

(e)

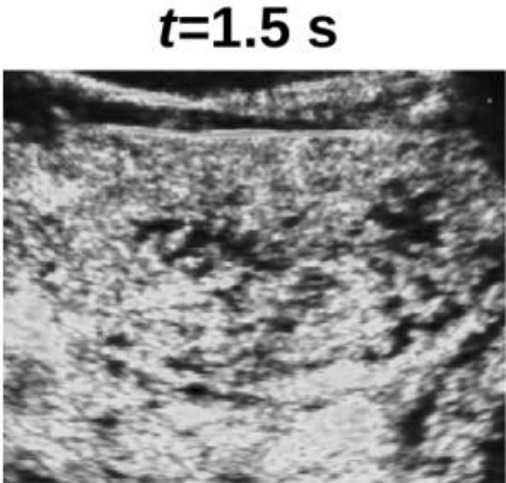

(c)

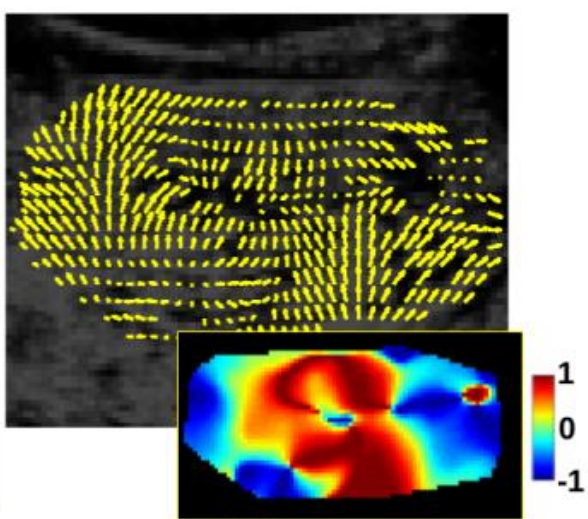

(f)

Fig. 2. Typical results from a patient with an I-HCA lesion. The manually drawn mask encompassing the lesion is shown in (a). Data obtained at different times during CEUS are shown: $0.5 \mathrm{~s}$ (left column), $1 \mathrm{~s}$ (middle column), and $1.5 \mathrm{~s}$ (right column) after bolus arrival. Contrast images (top row) and estimated, apparent vector transport fields (bottom row). The flow field is hyper-enhanced in the arterial phase (enhancement commences peripherally) and exhibits rapid centripetal filling. The pixel-wise centripetal indicator is shown as insets in the bottom row (note the large positive values, attributable to centripetal filling of the lesion). 


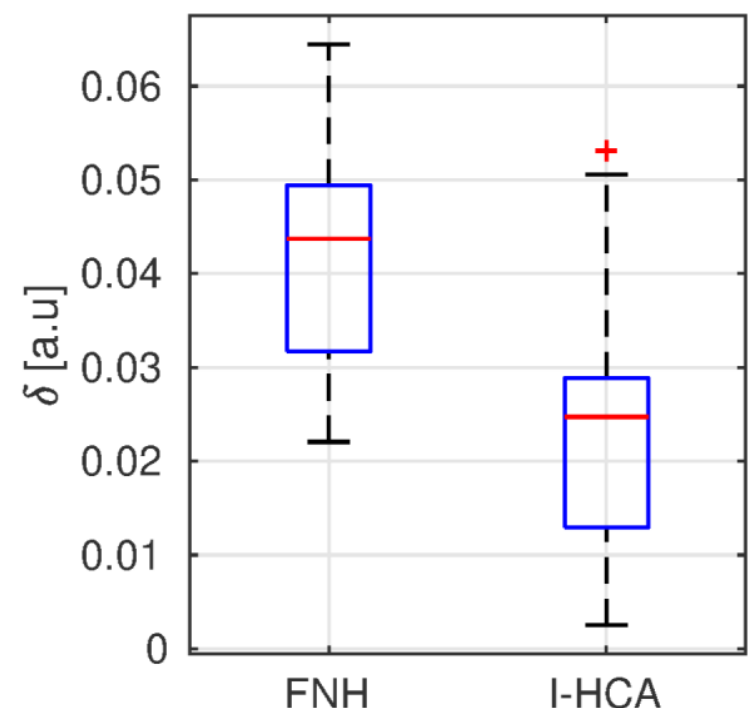

(a)

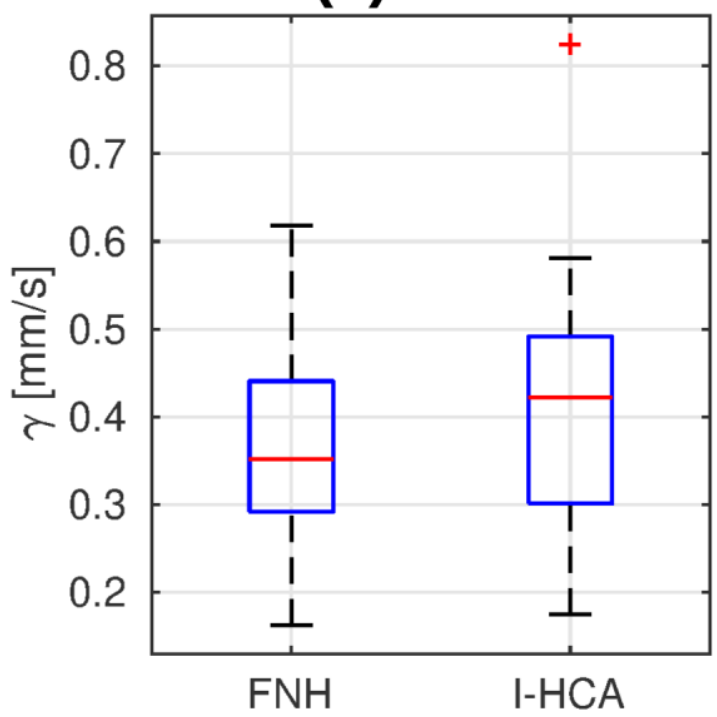

(c)

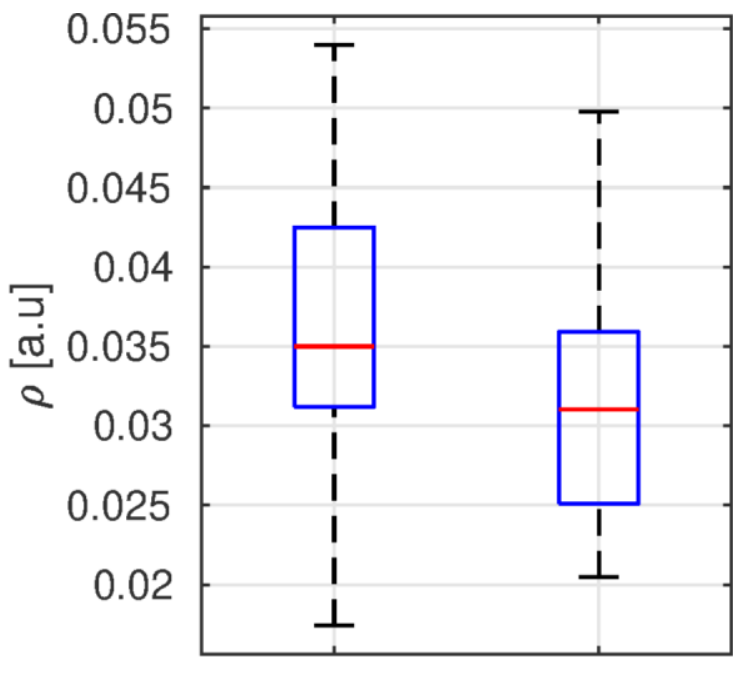

$\mathrm{FNH} \quad \mathrm{I}-\mathrm{HCA}$

(b)

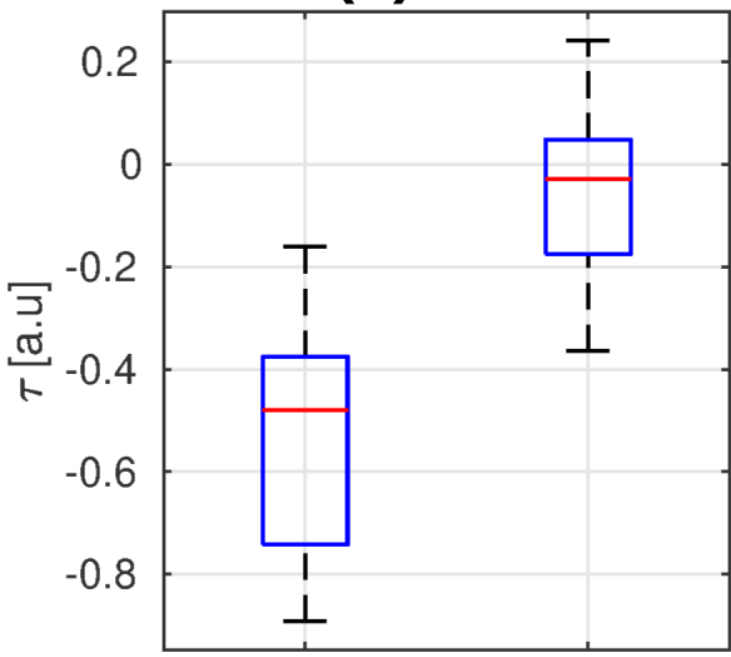

$\mathrm{FNH} \quad \mathrm{I}-\mathrm{HCA}$

(d)

Fig. 3. Boxplots of indicators of the four dense transport fields (divergence $\delta$ [a], curl $\rho[\mathrm{b}]$, amplitude $\gamma$ [c], and centripetal indicator $\tau$ [d]) for both patient populations (FNH vs. I-HCA selfvalidations). The medians are shown by the central marks, the first and third quartiles are the edges of the boxes, the whiskers extend to the most extreme timepoints not considered to be outliers, and the outliers are individually marked in red. 


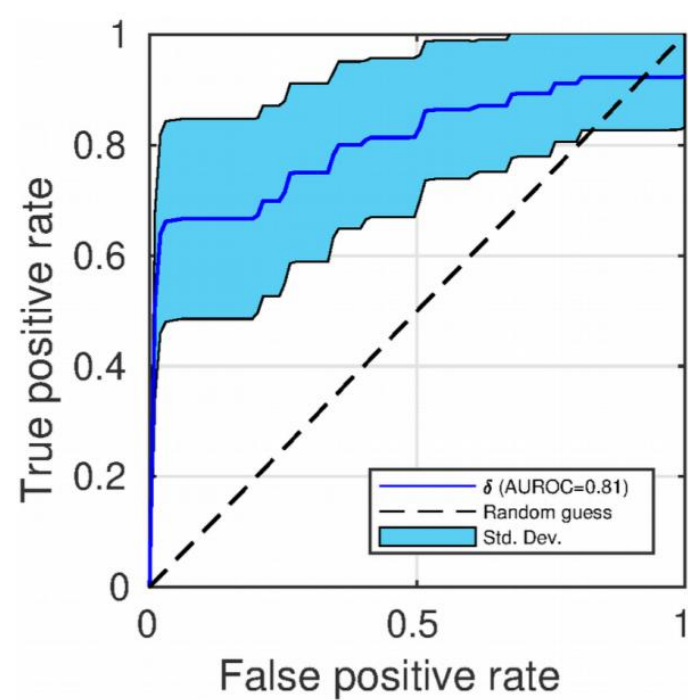

(a)

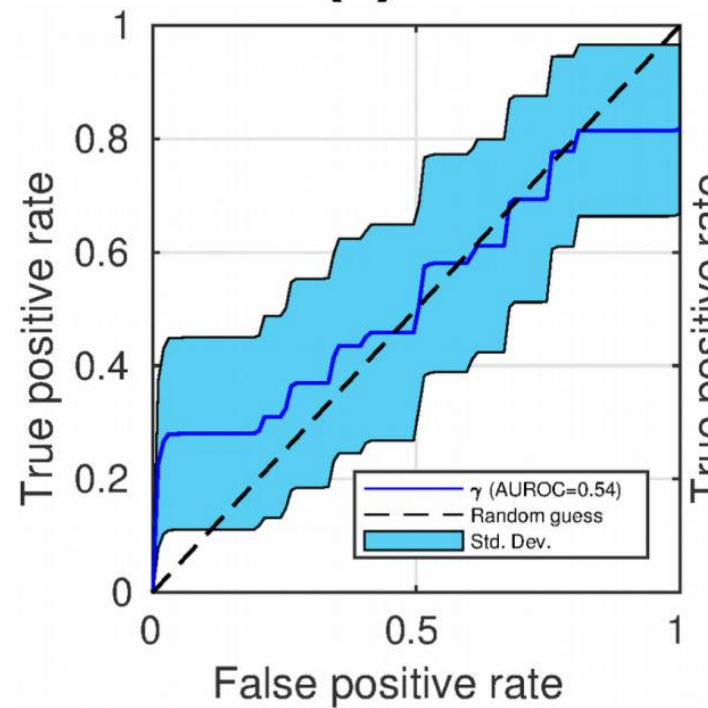

(c)

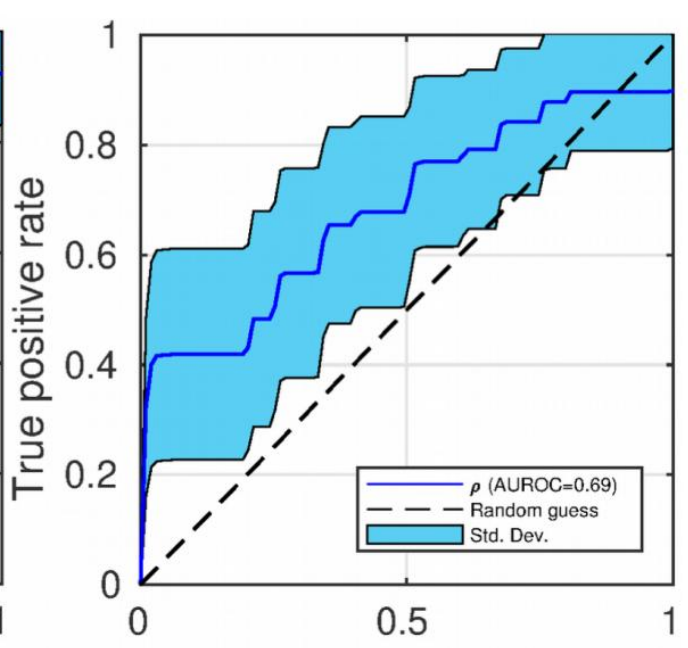

False positive rate

(b)

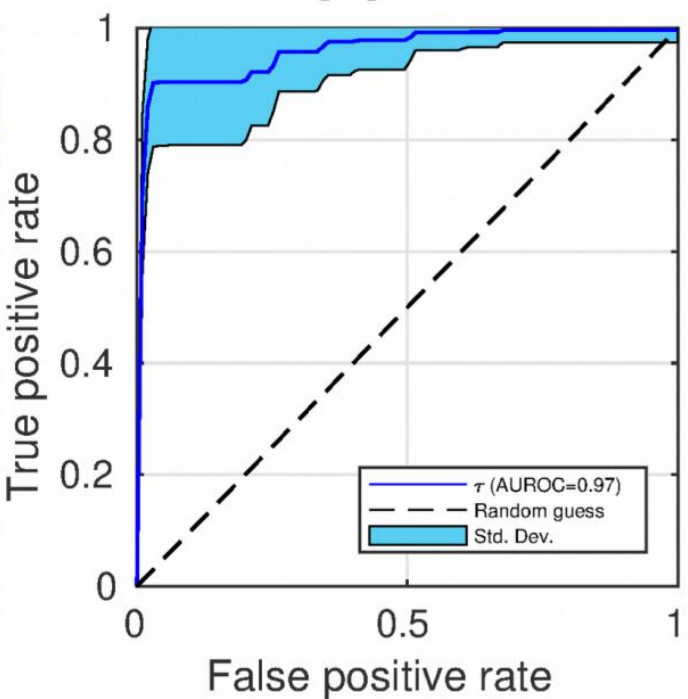

(d)

Fig. 4. ROC curves obtained using the four quantitative indicators (divergence $\delta$ [a], curl $\rho$ [b], amplitude $\gamma[\mathrm{c}]$, and centripetal indicator $\tau[\mathrm{d}]$ ) as binary classifiers (naive Bayes) for the two populations (i.e., FNH vs. I-HCA) after 10-fold cross-validation. 


\section{APPENDIX 1: Estimation of apparent microbubble displacement during CEUS}

This appendix deals with numerical implementation of the algorithm estimating microbubble displacement during CEUS. For each CEUS clip, the lesion was first manually delineated on a hyper-enhanced image. A binary mask was then constructed (this is termed $\Gamma$ below). We then proceeded as follows:

\section{Estimation of CEUS dense transport fields}

We used the transport equation to estimate the apparent microbubble transport field $(\vec{V})$, as suggested in (15):

$I_{t}+\vec{V} \cdot \vec{\nabla} I$

where $I$ denotes the grey level intensity on CEUS images and $I_{t}$ the partial temporal derivative of $I$. Practically, the desired transport field $V$ was estimated between two points in time $(t$ and $t+\delta t$, respectively) on a pixel-by-pixel basis using the so-called "optical flow" process (17). The algorithm yields the displacement between two images when the following function is optimised (19):

$\operatorname{argmin}_{\vec{V}} \int_{\Omega}\left|I_{t}+\vec{V} \cdot \vec{\nabla} I\right|+\alpha\left(\|\vec{\nabla} u\|_{2}^{2}+\|\vec{\nabla} v\|_{2}^{2}\right) d \vec{r}$

where $\Omega \in R e^{2}$ is the image-coordinate domain, $(u, v)$ the estimated pixel-wise transport vector components, and $\vec{r} \in \Omega$ the spatial location. All slices were re-sampled via bi-cubic interpolation to obtain a common isotropic, in-plane, $0.25 \times 0.25-\mathrm{mm}^{2}$ pixel representation. A spatial low-pass filter was then applied (29) (the cut-off frequency was the proportional pixel fraction of the original image, divided by 16, as suggested by (15)) to mitigate the impact of US speckles on the transport equation. An in-house developed, freely available, software provided 2D transport fields using the optical flow metric of Eq. (2) (http://bsenneville.free.fr/RealTITracker/). The reader is referred to (15) for additional details on the numerical implementation of Eq. (2).

Note that possible periodic, spontaneous, and drift displacements of tissue must be initially compensated for (24)(25), because they may change image intensities over time; Eq. 1 would (erroneously) attribute such changes to microbubble transport. As proposed in (15), B-mode 
images, which are not prone to contrast enhancement, are used to this end. We estimated translational displacements restricted to the binary mask $\Gamma$. We used a gradient-driven descent algorithm maximising the inter-correlation coefficients. This translation was used to compensate for displacement of imaged tissues on CEUS images prior to microbubble transport estimation employing Eq. (2).

\section{Pixel-wise analysis of dense flow fields}

We next calculated a pixel-wise understanding of flow directions/orientations and amplitude, as follows:

Maps of sources and sinks: Sources and sinks in the transport were analysed using the divergence operator. Mathematically, the divergence of a two-dimensional vector $\vec{V}=(u, v)$ is expressed as:

$\operatorname{div} \vec{V}=\vec{\nabla} \cdot \vec{V}=\frac{\partial u}{\partial x}+\frac{\partial v}{\partial y}$

The final, discrete divergence operator employed in numerical implementation was:

$(\operatorname{div} \vec{V})_{i, j, t}=u_{i+1, j, t}-u_{i, j, t}+v_{i, j+1, t}-v_{i, j, t}$

where $(i, j) \in \Omega$ denotes the pixel coordinates and $t$ the frame acquisition time. We emphasise that positive and negative values are associated with pixels located near sources and sinks, respectively.

Vortex maps: Local vortices in the estimated transport vectors were analysed with the aid of the curl operator. Mathematically, the curl of $\vec{V}$ is expressed as:

$\operatorname{curl} \vec{V}=\vec{\nabla} \Lambda \vec{V}=\frac{\partial v}{\partial x}-\frac{\partial u}{\partial y}$

The resulting discrete curl operator is:

$(\operatorname{curl} \vec{V})_{i, j, t}=v_{i+1, j, t}-v_{i, j, t}+u_{i, j+1, t}-u_{i, j, t}$

Amplitude maps: The amplitudes of estimated transport vectors were calculated as Euclidian distances. Mathematically, the magnitude of $\vec{V}$ is expressed by:

$\|\vec{V}\|_{2}=\sqrt{u^{2}+v^{2}}$ 
Centripetal indicator maps: The convergence of estimated transport vectors towards the centre of tumour were calculated with the aid of the scalar product. The cosine of the angle formed by two vectors $\vec{V}$ and $\vec{W}$ is expressed by:

$\cos (\widehat{\vec{V}, \vec{W}})=\frac{\vec{V} \cdot \vec{W}}{\|\vec{V}\|_{2}\|\vec{W}\|_{2}}$

In our study, $\vec{V}$ is any vector of the estimated flow field, and $\vec{W}$ has the same origin as $\vec{V}$, but the extremity located at the gravity centre of tumour (i.e, the centre of mass of the binary mask $\Gamma$ ). That way, the cosine of the angle $(\overrightarrow{\vec{V}, \vec{W}})$ lies in intervals $[-1,0]$ and $[0,1]$ for centrifugal and centripetal $\vec{V}$, respectively.

\section{Quantitative analysis of dense flow fields}

As described above, we created four sets of pixel-wise maps (of divergence, curl, amplitude and convergence towards the centre of tumour). Each set was then simplified to a single parameter as follows. The spatiotemporal averages of each map were individually computed under a mask defining the imaged tissue (i.e., $\Gamma$ ) within the relevant time window. The duration spanned by that window is termed $\Delta T$ below and commenced at the bolus arrival time $t_{0}$. Spatiotemporal averaging was weighted by the grey level intensity in CEUS image $I$; thus, the values for scenarios exhibiting identical microbubble transport behaviours were identical irrespective of the numbers of pixels evidencing microbubbles. The divergence and curl operators were termed $\delta$ and $\rho$, respectively. We measured the absolute value of curl; thus, the direction of vortex rotation didn't affected analysis. The centripetal indicator was termed $\tau$.

$$
\begin{gathered}
\delta=\frac{\sum_{i, j, t} I_{i, j, t}(\nabla \cdot \vec{V})_{i, j, t}}{\sum_{i, j, t} I_{i, j, t}} \\
\rho=\frac{\sum_{i, j, t} I_{i, j, t}\left|(\nabla \Delta \vec{V})_{i, j, t}\right|}{\sum_{i, j, t} I_{i, j, t}} \\
\gamma=\frac{\sum_{i, j, t} I_{i, j, t}\left\|\vec{V}_{i, j, t}\right\|_{2}}{\sum_{i, j, t} I_{i, j, t}}
\end{gathered}
$$


$\tau=\frac{\sum_{i, j, t} I_{i, j, t} \cos \left(\vec{V}_{l, j, t} \widehat{\vec{W}}_{l, j, t}\right)}{\sum_{i, j, t} I_{i, j, t}}$

with:

$\left\{\begin{array}{c}t \in\left[t_{0}, t_{0}+\Delta T\right] \\ \quad(i, j) \in \Gamma\end{array}\right.$

\section{Determination of the temporal window}

The temporal window of analysis was of duration $\Delta T$ and commenced at the bolus arrival time $t_{0}$; this was determined individually for each patient. To this end, we used a published time intensity curve (TIC) widely employed to determine time constants (10). The average US image intensity over $\Gamma$ (termed $\bar{I}_{\Gamma}(t)$ ) was analysed as a function of time using a two-compartment model, as follows:

$\bar{I}_{\Gamma}(t)=I_{\infty}\left[1-e^{-k\left(t-t_{0}\right)}\right]$

where $I_{\infty}$ is the asymptotic US signal enhancement, and $k$ the uptake rate. $I_{\infty}, t_{0}$, and $k$ were computed using the Levenberg-Marquardt least-square fit (30) employing all images of the US sequence. The use of a simple two-compartment model was motivated by the fact that only the rise step was screened. The goodness-of-fit was considered acceptable when the Pearson correlation coefficient $\left(r^{2}\right)$ was $>0.95$. In such cases, the $t_{0}$ values chosen earlier served as the start times for the temporal windows.

\section{Hardware and implementation}

Our test platform was an Intel $2.5 \mathrm{GHz}$ i7 workstation (eight cores) with $32 \mathrm{~GB}$ of RAM. The implementation was performed in $\mathrm{C}++$ and parallelised via multi-threading. 
The English in this document has been checked by at least two professional editors, both native speakers of English. For a certificate, please see: 